\title{
GRÈZES-RUEFF (François), LEDUC (Jean), Histoire des élèves en France. De l'Ancien Régime à nos jours
}

Paris : Armand Colin, 2007. 451 p.

\section{Marcel Grandière}

\section{OpenEdition}

Journals

\section{Édition électronique}

URL : https://journals.openedition.org/histoire-education/531

DOI : 10.4000/histoire-education.531

ISSN : 2102-5452

\section{Éditeur}

ENS Éditions

\section{Édition imprimée}

Date de publication : 1 avril 2008

Pagination : 139-142

ISBN : 978-2-7342-1117-4

ISSN : 0221-6280

\section{Référence électronique}

Marcel Grandière, « GRÈZES-RUEFF (François), LEDUC (Jean), Histoire des élèves en France. De l'Ancien Régime à nos jours », Histoire de l'éducation [En ligne], 118 | 2008, mis en ligne le 16 octobre 2008, consulté le 20 mai 2021. URL : http://journals.openedition.org/histoire-education/531 ; DOI : https:// doi.org/10.4000/histoire-education.531

Ce document a été généré automatiquement le 20 mai 2021.

(ㄷ) Tous droits réservés 


\title{
GRÈZES-RUEFF (François), LEDUC (Jean), Histoire des élèves en France. De l'Ancien Régime à nos jours
}

Paris : Armand Colin, 2007. 451 p.

\author{
Marcel Grandière
}

\section{RÉFÉRENCE}

GRÈZES-RUEFF (François), LEDUC (Jean), Histoire des élèves en France. De l'Ancien Régime à nos jours, Paris : Armand Colin, 2007. $451 \mathrm{p}$.

1 C'est une histoire ambitieuse que tentent ici les auteurs : faire voir la vie quotidienne des élèves des enseignements primaire et secondaire (selon la terminologie d'aujourd'hui) depuis l'Ancien Régime - mais seul, quasiment, le XVIIIe siècle est en fait concerné, souvent de manière anecdotique - jusqu'à 2006. L'étude est centrée sur la "vie scolaire », selon une expression apparue dans les textes de l'Éducation nationale en 1942 (p. 4). Les auteurs limitent ainsi leur sujet, et ne mettent pas au cœur de leurs préoccupations les contenus et les méthodes d'enseignement, pas plus que l'analyse sociologique des populations scolaires.

Limiter un tel sujet se comprend aisément, mais comment traiter de la vie quotidienne des élèves sans prendre en compte ce qui fait l'essentiel de leur existence de chaque jour : leur métier d'élèves ? L'organisation pédagogique, la manière dont sont conçus et organisés les apprentissages conditionnent la vie quotidienne, quelle que soit la période considérée. Les élèves d'Ancien Régime vivent au rythme des processus d'apprentissage mis en œuvre dans leurs collèges et de l'organisation du temps de la journée qui en résulte: deux séances de travail chaque jour avec le professeur, de deux heures et demie chacune le plus souvent, des devoirs quotidiens écrits et oraux importants, qui font des élèves des acteurs de leur formation. Les cours proprement dits, ou prélections, n'occupent qu'une partie des séances professorales, qui commencent par la répétition 
des leçons, la correction des devoirs, et s'achèvent par la dictée du "thème " ou " argument » qui va servir de sujet au devoir que doit faire l'élève pour acquérir les notions et les usages de la langue enseignée, le latin essentiellement.

C'est ainsi la conception des apprentissages qui joue sans doute le rôle le plus déterminant dans la vie quotidienne de l'élève. Ou bien celui-ci est longuement sollicité par ses devoirs écrits ou oraux, comme dans les collèges d'Ancien Régime, ce qui implique un équilibre entre les leçons professorales et le temps de travail personnel, alors solidement encadré pour les pensionnaires des collèges. Ou bien l'élève est surchargé de cours, comme cela commence à être le cas à la fin du XIXe siècle, ce qui réduit d'autant son autonomie - avec pas mal d'ambiguïté, souvent : c'est, par exemple, au moment où Jules Ferry demande davantage de liberté pour les élèves des écoles normales d'instituteurs et critique sévèrement la surcharge de travail qui leur est imposée que les nouveaux programmes de 1881 ajoutent encore de la matière à leur curriculum, aboutissant à un emploi du temps totalement rempli... Le mouvement pédagogique du XXe siècle tend formellement à mettre l'élève au cœur des apprentissages, au centre des processus d'acquisition. En quoi l'émergence des pédagogies nouvelles qui métamorphosent l'élève en « apprenant ", notamment dans le cadre de l'école primaire, a-t-elle transformé - ou non - la vie quotidienne des élèves ? À cette question, le livre n'apporte pas vraiment de réponse. Le choix exprimé d'emblée de ne pas mettre la pédagogie au cœur du sujet fige quelque peu les perspectives.

4 La démarche suivie par les auteurs compartimente le sujet de manière assez classique. Ils procèdent selon un plan thématique, en cinq parties : "Temps et espaces scolaires ", p. 47-135 ; «La discipline», p. 137-227 ; «Figures d'élèves », p. 229-295 ; «Rites de passage », p. 297-329; «La société des élèves », p. 331-404, tout cela après un chapitre d'introduction, "Le réquisitoire», consacré à la permanence de l'expression de rancœurs vis-à-vis de l'école, selon le témoignage tardif d'écrivains connus et d'auteurs de Mémoires. La conclusion est un essai de périodisation de cette histoire des élèves.

Nous savons que l' "édifice " scolaire est en constante discussion et reconstruction, quels que soient ses différents éléments : espaces scolaires, emploi du temps, conduite des élèves, organisation de leur travail... Sans doute pour mieux servir le même idéal, toujours rêvé : une belle éducation du cœur et de l'esprit, et aussi du corps. Au sujet de cette difficile mise en musique de la vie des élèves, les auteurs de cette histoire insistent avec raison sur l'importance de la seconde moitié du XIXe siècle (sans exclure le Second Empire, souvent oublié dans l'historiographie française), époque certainement décisive pour l'organisation générale des enseignements primaire et secondaire. Les évolutions de la vie des élèves au XXe siècle sont également mises en relief. Il faudrait toutefois mieux marquer l'effort d'organisation de la vie des écoliers entrepris au XVIIIe siècle. Les petites écoles connaissent alors une mise en ordre sans précédent, comme en témoignent les méthodes et règlements, souvent rédigés sous contrôle épiscopal, qui tentent de "mettre en règle " la vie des écoliers : disposition matérielle des salles, rituels d'entrée, distribution des élèves en bandes sous la conduite d'officiers ou d'officières, qui jouent un rôle important dans les classes, «ordre du temps » (journalier, hebdomadaire et annuel), ordre des gestes et attitudes, sous le terme de «modestie », manières de conduire les élèves, etc. C'est aussi au XVIIIe siècle que les pensions et maisons d'éducation privées se multiplient en ville pour accueillir les enfants des milieux aisés, aussi loin que possible des collèges. Derrière la clôture de ces maisons, les pédagogues novateurs mettent en place pour les élèves de nouveaux 
modes de vie, censés être plus conformes à la philosophie du siècle, issue des travaux de Locke. Malgré les vives protestations des professeurs des collèges publics, les familles sont attirées par ces institutions nouvelles. Même la qualité des repas répond aux vœux des parents, dès avant la Révolution donc, contrairement à ce qu'affirme le livre (p. 111 et suivantes): les nombreux prospectus de ces maisons précisent les menus, ainsi que les précautions prises quant à la santé des élèves. Le XIXe siècle n’a pas tout inventé.

6 Ainsi la vie des écoliers a-t-elle sans doute bien changé au XVIIIe siècle, ce que ne traduit pas le sous-titre "L'ancien régime disciplinaire » (p. 168), qui fait penser à une période d'horreur pour les élèves. Est-ce bien le cas? Les élèves d'Ancien Régime sont certes sous la menace des châtiments corporels, mais dans les grands collèges, ceux-ci ont tendance à s'atténuer dans la deuxième moitié du siècle ; à Angers, les Oratoriens ne les utilisent plus, comme le remarquent avec satisfaction les écoliers venant des autres collèges de la province, habitués à plus rude traitement dans leur petit pays ! Surtout, les externes, généralement majoritaires parmi les écoliers, jouissent finalement d'une relative liberté : peu d'heures de classe et logement en ville, chez leurs parents ou des logeurs. Pas de quoi faire des cauchemars tels que les racontent parfois des souvenirs d'école tardifs. Quant aux maisons d'éducation, elles affichent leur volonté de traiter avec douceur leurs pensionnaires : les familles y sont sensibles, et c'est un atout pour attirer des élèves.

7 C'est qu'une importante mutation s'opère à partir des premières décennies du siècle et s'accélère après 1750, sans guère être relevée dans cet ouvrage: la vague d'enfermement des jeunes gens dans des pensions privées et des maisons d'éducation. Les collèges publics se vident, d'où leur tendance à installer des pensions dans leurs murs pour freiner la baisse des effectifs, malgré le peu de goût des professeurs congréganistes pour cette tâche : ceux-ci résistent souvent le plus longtemps possible. Le modèle de l'internat à la mode, ce sont les écoles militaires créées par le comte de Saint-Germain en 1776. C'est un changement considérable, qui va se poursuivre et s'épanouir pendant la plus grande partie du XIXe siècle, pour s'éteindre finalement au siècle suivant, malgré quelques tentatives récentes pour revenir en arrière. C'est dans ces internats que les mauvais traitements, ou tout simplement la violence que représente pour de petits élèves la séparation d'avec la famille, ont pu le mieux s'exercer. Cet enfermement aurait mérité d'être pris en compte dans les essais de périodisation proposés ici.

8 Une remarque de méthode enfin. Le livre utilise beaucoup, et c'est bien normal, les témoignages littéraires et mémoires, qui relatent la plupart du temps les mauvais souvenirs de la scolarité. Même si les auteurs affichent leur recul par rapport à ces témoignages, ces derniers paraissent quand même «faire histoire » par la facilité même de les recueillir et la force d'expression qu'ils véhiculent. Ils ne traduisent le plus souvent que l'image que les narrateurs cherchent à donner d'eux-mêmes à la fin de leur vie, sans en avoir vraiment conscience peut-être. Est-ce la raison pour laquelle certains chapitres paraissent peu copieux, comme celui relatif à «La camaraderie» (p. 333-360) ? De Rabelais, Rousseau, Ferry, aux pourfendeurs actuels du système éducatif, les plaintes contre le mauvais fonctionnement de l'école sont toujours aussi vives : pour réformer, il faut toujours commencer par critiquer sévèrement l'existant, ce qui constitue une vraie difficulté pour les historiens. Un fait récent vient contredire ces plaintes: malgré tout ce qu'on peut lire encore aujourd'hui, les Français se 
montrent satisfaits de leur école... à $88 \%$ des plus de 15 ans, selon le dernier sondage effectué en 2007 ! Leur vie d'élèves ne leur pèse pas trop. Les mémoires ne disent pas tout.

\section{AUTEURS}

\section{MARCEL GRANDIÈRE}

